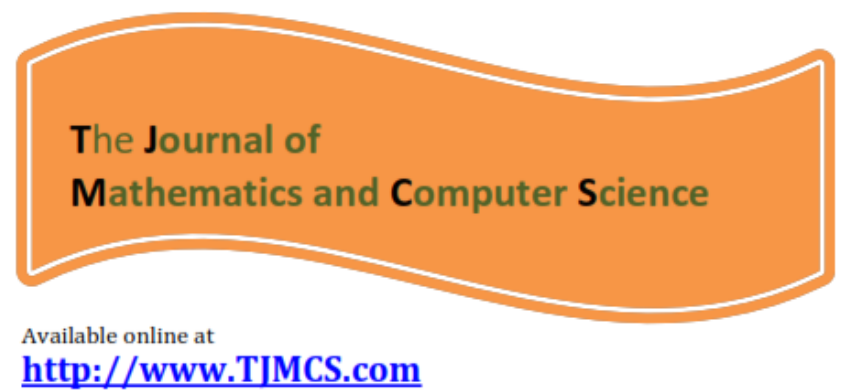

The Journal of Mathematics and Computer Science Vol. 4 No.2 (2012) 278 - 282

\title{
Explicit Analytical Solution for a kind of Time-Fractional Evolution Equations by He's Homotopy Perturbation Methods
}

\author{
G.A. Afrouzi *, 1 , R.A. Talarposhti *, H. Ahangar * \\ * Department of Mathematics, Faculty of Mathematical Sciences, University of \\ Mazandaran, Babolsar, Iran
}

Received: January 2012, Revised: April 2012

Online Publication: June 2012

\begin{abstract}
In this letter, a kind of powerful analytical method, called Homotopy-perturbation method (HPM) is introduced to obtain the exact solutions for a kind of evolution equations with fractional derivatives. These initial value problems have significant importance in applied science and many field of engineering. In this method, a nonlinear complex differential equation is transformed to a series of linear and nonlinear parts, almost simpler differential equations. These sets of equations are then solved iteratively. finally, a linear series of the solutions completes the answer if the convergence is maintained. The results show that the proposed method is very efficient and convenient and can readily be applied to a large class of fractional problems.
\end{abstract}

Keywords.Homotopy-perturbation method (HPM); exact solution; Initial value problems (IVP); Fractional evolution equations.

AMS (MOS) subject classification:

\section{Introduction}

In recent years considerable interest in fractional differential equations (FDE) has been stimulated due to their numerous applications in the areas of physics and engineering. Recently, some promising approximate analytical solutions are proposed for solving different kinds of linear and nonlinear problems. Variational iteration method is relatively new approaches to provide analytical approximations to linear and nonlinear equations. For the first time, Ji-Huan He [1] applied VIM to fractional differential equations. Recently, Odibat and Momani [2] implemented the method to solve nonlinear ordinary differential equations of fractional order. The evolution equations arise in many modeling problems in physics and hydromechanics. In this study, He's homotopy perturbation method [3] is implemented to obtain exact analytical solution for a kind of evolution equations with fractional order. This method does not depend on a small parameter. Using homotopy technique in topology, a homotopy is constructed with an embedding parameter , which is considered as a "small parameter". HPM has been shown to effectively, easily and accurately solve a large class of linear and nonlinear problems with components converging rapidly to accurate solutions.

\footnotetext{
${ }^{1}$ Corresponding author: Tel/Fax: +98 1125242003 E-mail address: afrouzi@umz.ac.ir
} 


\section{Basic definitions}

Definition 2.1. A real function $f(x), x>0$, is said to be in the space $C_{\alpha}, \alpha \in R$ if there exists a real number $p(>\alpha)$, such that $f(x)=x^{p} f_{1}(x)$, where $f 1(x) \in C[0, \infty)$. Clearly $C_{\alpha} \subset C_{\beta}$ if $\beta \leq \alpha$.

Definition 2.2. function $f(x), x>0$, is said to be in the space $C_{\alpha}^{m}, m \in$ $N \cup 0$, if $f^{(m)} \in C_{\alpha}$.

Definition 2.3. For $m$ to be the smallest integer that exceeds $\mu$, the Caputo time-fractional derivative operator of order $\mu>0$ is defined as

$$
\begin{aligned}
& D^{\mu} u(x, t)= \\
& \quad \frac{\partial^{\mu} u(x, t)}{\partial t^{\mu}}= \begin{cases}\frac{1}{\Gamma(m-\mu)} \int_{0}^{t}(t-\tau)^{m-\mu-1} \frac{\partial^{m} u(x, \tau)}{\partial \tau^{m}} d \tau, & m-1<\mu \leq m, \\
\frac{d^{m}}{d t^{m}} u(x, t), & \mu=m .\end{cases}
\end{aligned}
$$

For more information on the mathematical properties of fractional derivatives and integrals, one can consult the mentioned references.

\section{Homotopy-perturbation method}

In this letter, we apply the Homotopy-perturbation method to the discussed problems. To illustrate the basic ideas of the new method, we consider the following nonlinear differential equation,

$$
A(u)-f(r)=0, \quad r \in \Omega,
$$

with the boundary condition of:

$$
B\left(u, \frac{\partial u}{\partial n}\right)=0, \quad r \in \Gamma,
$$

where $A$ is a general differential operator, $B$ a boundary operator, $f(r)$ a known analytical function and $\Gamma$ is the boundary of the domain $\Omega . A(u)$ is defined as follows:

$$
A(u)=L(u)+N(u) .
$$

Homotopy-perturbation structure is shown as:

$$
H(v, p)=L(v)-L\left(u_{0}\right)+p L\left(u_{0}\right)+p[N(v)-f(r)]=0,
$$

or

$$
H(v, p)=(1-p)\left[L(v)-L\left(u_{0}\right)\right]+p[A(v)-f(r)]=0,
$$


where,

$$
v(r, p): \Omega \times[0,1] \longrightarrow \mathbb{R} .
$$

Obviously, considering Eqs. (5) and (6) we have:

$$
H(v, 0)=L(v)-L\left(u_{0}\right)=0, \quad H(v, 1)=A(v)-f(r)=0,
$$

where $p \in[0,1]$ is an embedding parameter and $u_{0}$ is the first approximation that satisfies the boundary condition. The process of the changes in $p$ from zero to unity is that of $v(r, p)$ changing from $u_{0}$ to $u_{r}$. We consider $v$ as:

$$
v=v_{0}+p v_{1}+p^{2} v_{2}+\ldots,
$$

and the best approximation is:

$$
u=\lim _{p \longrightarrow 1} v=v_{0}+v_{1}+v_{2}+\ldots
$$

\section{Modified homotopy perturbation method}

Recently, Shaher Momani [4] applied homotopy perturbation method to fractional differential equations. To illustrate the basic ideas of the new modification, we consider the following nonlinear differential equation of fractional order:

$$
D^{\mu} u(t)+L(u(t))+N(u(t))=f(t), \quad t>0, \quad m-1<\mu \leq m,
$$

where $L$ is a linear operator which might include other fractional derivatives of order less than $\mu, N$ is a nonlinear operator which also might include other fractional derivatives of order less than $\mu, f$ is a known analytic function and $D^{\mu}$ is the Caputo fractional derivative of order $\mu$, subject to the initial conditions

$$
u^{(k)}(0)=c_{k}, \quad k=0,1,2, \ldots, m-1 .
$$

In view of the homotopy technique, we can construct the following homotopy:

$$
u^{(m)}-f(t)=p\left[u^{(m)}-L(u)-N(u)-D^{\mu} u\right], \quad p \in[0,1] .
$$

\section{$5 \quad$ Numerical example}

Example 5.1. We finally consider fifth-order Kdv equation with fractional derivative.

$$
\frac{\partial^{\alpha} u(x, t)}{\partial t^{\alpha}}+u(x, t) \frac{\partial u(x, t)}{\partial x}-u(x, t) \frac{\partial^{3} u(x, t)}{\partial x^{3}}-\frac{\partial^{5} u(x, t)}{\partial x^{5}}=0
$$


subject to the initial condition of:

$$
u(x, 0)=e^{x} .
$$

In order to solve Eq. (14) using HPM, we construct the following homotopy:

$$
\begin{gathered}
\frac{\partial u(x, t)}{\partial t}=p\left[\frac{\partial u(x, t)}{\partial t}-u(x, t) \frac{\partial u(x, t)}{\partial x}+u(x, t) \frac{\partial^{3} u(x, t)}{\partial x^{3}}\right. \\
\left.+\frac{\partial^{5} u(x, t)}{\partial x^{5}}-\frac{\partial^{\alpha} u(x, t)}{\partial t^{\alpha}}\right]
\end{gathered}
$$

Substituting $u$ from Eq. (9) into Eq. (16) and rearranging based on powers of $p$-terms, we can obtain:

$$
\begin{gathered}
p^{0}:\left\{\frac{\partial u_{0}(x, t)}{\partial t}=0,\right. \\
p^{1}:\left\{\frac{\partial u_{1}(x, t)}{\partial t}=\frac{\partial u_{0}(x, t)}{\partial t}-u_{0}(x, t) \frac{\partial u_{0}(x, t)}{\partial x}\right. \\
+u_{0}(x, t) \frac{\partial^{3} u_{0}(x, t)}{\partial x^{3}}+\frac{\partial^{5} u_{0}(x, t)}{\partial x^{5}}-\frac{\partial^{\alpha} u_{0}(x, t)}{\partial t^{\alpha}}, \\
p^{2}:\left\{\frac{\partial u_{2}(x, t)}{\partial t}=\frac{\partial u_{1}(x, t)}{\partial t}-u_{1}(x, t) \frac{\partial u_{1}(x, t)}{\partial x}\right. \\
+u_{1}(x, t) \frac{\partial^{3} u_{1}(x, t)}{\partial x^{3}}+\frac{\partial^{5} u_{1}(x, t)}{\partial x^{5}}-\frac{\partial^{\alpha} u_{1}(x, t)}{\partial t^{\alpha}},
\end{gathered}
$$

and therefore,

$$
\begin{gathered}
u_{0}(x, t)=e^{x} \\
u_{1}(x, t)=e^{x} t \\
u_{2}(x, t)=\frac{1}{2} e^{x} t^{2}-\frac{e^{x} t^{(2-\alpha)}}{\Gamma(2-\alpha)(2-\alpha)}+e^{x} t \\
\vdots
\end{gathered}
$$

The solution of this equation, when $p \rightarrow 1$, will be as follows:

$$
u(x, t)=u_{0}(x, t)+u_{1}(x, t)+u_{2}(x, t)+u_{3}(x, t) .
$$


Substituting $\alpha=1$ into $u(x, t)$, we finally obtain the solution as follows:

$$
u(x, t)=e^{x} \sum_{n=0}^{\infty} \frac{t^{n}}{n !}=e^{x} e^{t}=e^{x+t} .
$$

which is the exact solution of Eq.(45)

\section{Conclusion}

In this paper,the homotopy perturbation method (HPM) has been successfully applied to study a kind of the time fractional evolution equations with variable coefficients. The solution obtained by means of the homotopy perturbation method is an infinite power series with respect to appropriate initial condition, which can be, in turn,expressed in a closed form. In this work, we used the Mathematical Package to calculate the series obtained by homotopy perturbation method. The results show that homotopy perturbation method is powerful and efficient techniques in finding exact and approximate solutions for linear inhomogeneous partial differential equations of fractional order in fluid mechanics. They provide more realistic series solutions that converge very rapidly in real physical problems. The implementation of the noise terms, if exist, is a powerful tool to accelerate the convergence of the solution. The obtained results reinforce the conclusions made by many researchers about the efficiency of the HPM.

\section{References}

[1] I. Podlubny, Fractional Differential Equations, Academic Press, San Diego, 1999.

[2] J.H. He, Approximate analytical solution for seepage flow with fractional derivatives in porous media, Computer Methods in Applied Mechanics and Engineering, 167 (1998), pp. 57-68.

[3] J.H. He, Approximate analytical solution for seepage flow with fractional derivatives in porous media, Comput. Methods Appl. Mech. Eng. 167 (1998), pp. 57-68.

[4] Z. Odibat, S. Momani, Application of Variational Iteration Method to Nonlinear Differential Equations of Fractional Order, International Journal of Nonlinear Science and Numerical Simulation, 7 (1) (2006): 27-34

[5] A. Sadighi and D.D. Ganji, Exact solutions of Laplace equation by homotopyperturbation and Adomian decomposition methods, Physics Letters A, Volume 367, Issues 1-2, 16 July 2007, Pages 83-87. 